\title{
Insulating Packaging Solution Based on Cylinder Model
}

\author{
Jing Qian ${ }^{1,2}$ and Yanbing Zhao ${ }^{2}$ \\ ${ }^{1}$ Department of Packaging Engineering, School of Mechanical Engineering, Jiangnan University, 1800 Lihu Avenue, \\ Wuxi, Jiangsu 214122, China \\ ${ }^{2}$ School of Mechanical Engineering, Jiangnan University, Wuxi, Jiangsu 214122, China
}

Correspondence should be addressed to Jing Qian; qj639@163.com

Received 7 March 2013; Revised 7 May 2013; Accepted 27 May 2013

Academic Editor: Changfeng Ge

Copyright (C) 2013 J. Qian and Y. Zhao. This is an open access article distributed under the Creative Commons Attribution License, which permits unrestricted use, distribution, and reproduction in any medium, provided the original work is properly cited.

Insulating packaging is used in a variety of applications as a thermal protection for temperature-sensitive products. With computer simulation, one spherical shell model and one cylinder model are identified as suitable result for insulating packaging solution. Based on the cylinder model, the insulating packaging solution is achieved by using "minimum packaging cost" as an optimization target. The comparison of results predicted from the present model with benchmark experimental data shows satisfactory agreement in terms of packaging duration.

\section{Introduction}

An insulating package can maintain product temperatures within acceptable ranges and slow the deterioration of product in the distribution environment until it reaches the consumer. A whole packaging system is comprised of an insulating container, phase change materials (PCM), and product. Using materials with low thermal conductivities in insulating packaging conserves energy by reducing heat loss or gain and by reducing temperature variation within products. Phase change materials (PCM), often called temperature stabilizers or refrigerant, can be formulated and conditioned at specific temperatures and can be used in concert with the product itself and the rest of the packaging to create an environment within the shipping container to thermally protect the product $[1,2]$.

Researchers have developed different mathematical models to predict the capability of the insulating package system which already exists. Based on the existing insulating package systems, investigators developed expressions of the insulating package system $R$-value (thermal resistances) for determining heat transfer on a one-dimensional plane surface and subsequently for predicting the quantity of PCM or the allowable shipping time which the insulating package system can provide under specific ambient temperature and desired product temperature [3-7]. However, before shipping a temperature-sensitive product, neither the interrelated dimension of an insulated container nor the quantity of PCM is usually known. All these parameters of the insulating package system need to be determined by the designer to successfully protect the product. In this research, by comparing spherical shell model and cylinder model on describing normal three-dimensional insulating packaging system, a mathematical model which could be a tool for packaging designer was developed, and insulating packaging solutions are achieved.

\section{Spherical Shell Model and Cylinder Model}

Real insulating containers have three-dimensional geometries. However, it is difficult to get a design solution for a three-dimensional model. In the research, spherical shell models and cylinder models were developed as a transformation tool for an insulating packaging system. Suppose the inside dimensions of an insulating container are $L \times W \times H \times$ $\Delta x$ (suppose $H>L>W$ ), where the thickness of container is $\Delta x$, as in Figures 1 and 2.

2.1. Spherical Shell Model. To realize the insulating packaging design, a spherical shell model has been developed (see Figure 1, (1) as followed). The spherical shell model in 


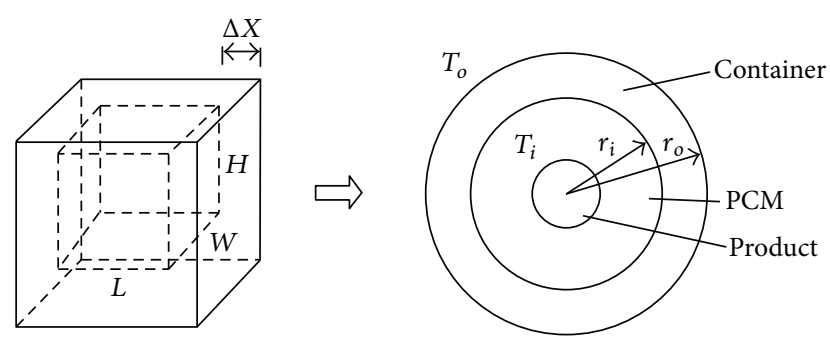

Figure 1: Transformational sketch from rectangular system to spherical shell model.

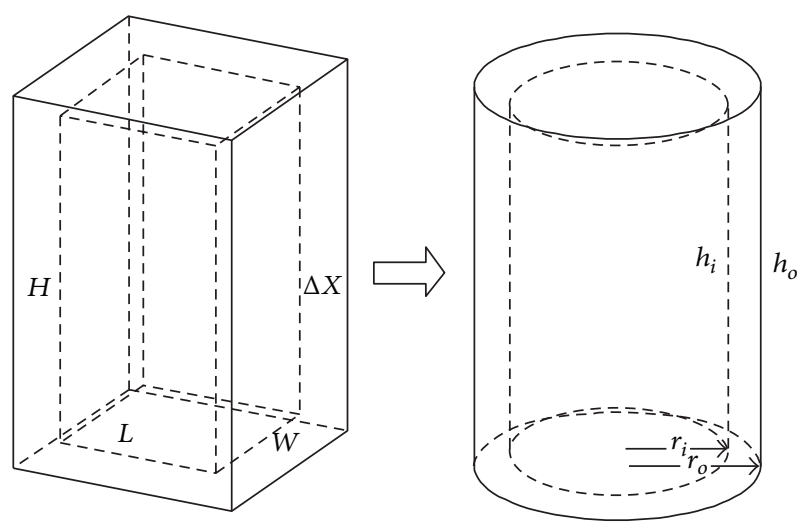

FIgURE 2: Transformational sketch from rectangular system to cylinder model.

this research has been confirmed by using finite element computer program and experiments [8]. This model is an appropriate tool to transfer a rectangular insulating container to a spherical shell in which the inner volume of spherical shell model equals the inner volume of the rectangular container and the thickness in one equals that in the other. Research shows that when the ratio of maximum and minimum side of rectangular container $(H / W)$ is not more than 2.5 , the conversion model has enough accuracy (the error of duration time is not more than 6\%; see Figure 3) for packaging design:

$$
\begin{gathered}
r_{i}=\sqrt[3]{\frac{L \cdot W \cdot H}{((4 / 3) \pi)}}, \\
r_{o}=r_{i}+\Delta x .
\end{gathered}
$$

2.2. Cylinder Model. When the ratio of sides is more than 2.5, a new conversion model was developed as a cylinder model (see Figure 2 and (2) and (3) as follows). The cylinder model with the same longest inner side of the container as the height of the model ( $h_{i}=H$ as in Figure 2$)$ was checked by ANSYS finite element simulations and tests. The best cylinder model

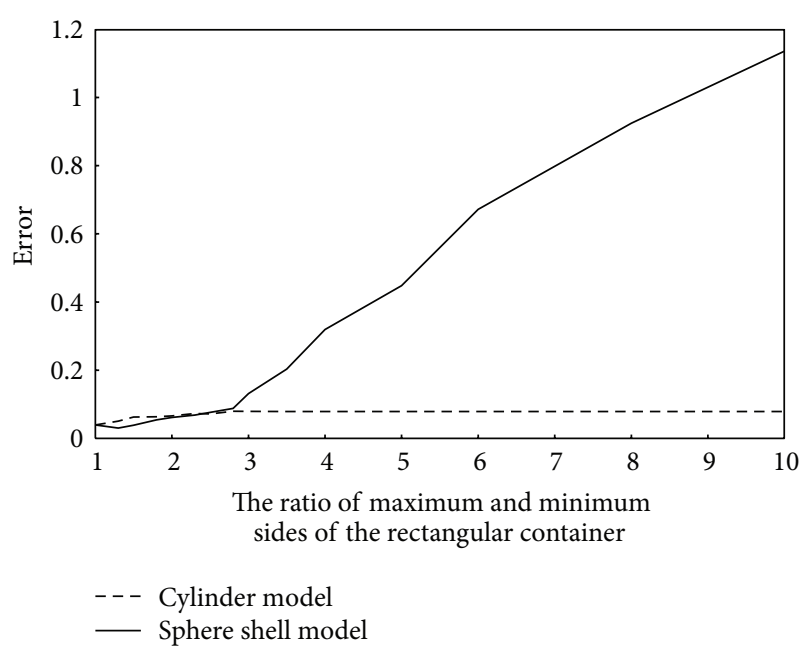

FIgURE 3: Errors between rectangular containers and different mathematical models.

is when its inner volume equals to inner volume of container and the thickness of one equals that of the other [9]:

$$
\begin{gathered}
r_{i}=\sqrt{\frac{L \times W}{\pi}}, \\
r_{o}=\sqrt{\frac{L \times W}{\pi}}+\Delta x .
\end{gathered}
$$

In Figure 3, the basic dimension is $200 \mathrm{~mm}$, and the thickness of container is $30 \mathrm{~mm}$. For example, when the ratio of maximum and Minimum. sides of rectangular container $(H / W)$ is 2 , the dimensions of real container are $200 \times 200 \times$ $400 \times 30 \mathrm{~mm}$. The error is a deviation of duration times between models and real insulating system.

The research shows that when the ratio of side increases, the errors of duration time of cylinder model are stabilized by no more than $10 \%$. Therefore, the cylinder model is a reasonable choice as a transformation tool to get insulating packaging solution. Hereafter the research works on development of mathematical heat transfer model based on cylinder model.

\section{Insulating Packaging Solution}

3.1. Heat Transfer Model Based on Cylinder Model. To simplify the basic models, approximating the real heat transfer through insulating package, it is assumed that

(1) a steady-state condition exists. The outside temperature of package is $T_{o}$; the inside temperature of package (target temperature) is $T_{i}$; the ambient temperature is $T_{\infty}$;

(2) the phase change material (PCM) is the main thermal sink to keep the temperature of the product constant or in a proper range;

(3) when the phase change happens, the temperature of PCM in the package is uniformly constant at its melting point; 
(4) duration of the package is the time that it takes for phase changing of the PCM in the research. Realistically, duration of the package is longer than this definition as the temperature of PCMs could be lower than the melting point, and the allowance temperature of product could be higher than the melting point for some of the products. In this case, sensible heat gain can be balanced through increasing the PCM temperature to provide for a longer shipping time.

When an insulating package is transported on a highway in summer, the inside temperature of the truck trailer can be over $60^{\circ} \mathrm{C}$. In this case, both convection and radiation should be considered. With the combination of convection and radiation on the cylinder model, the heat which transfers from outside to inside of the insulating package can be expressed by the following equation:

$$
\begin{aligned}
h_{a} \cdot A_{o} \cdot\left(T_{\infty}-T_{o}\right)+\varepsilon \sigma A_{o} \cdot\left(T_{\infty}^{4}-T_{o}^{4}\right) \\
=2 \pi \cdot k \cdot\left(T_{o}-T_{i}\right)\left(\frac{r_{o}^{2}}{\Delta x}+\frac{h_{o}}{\ln \left(r_{o} / r_{i}\right)}\right) \\
=\frac{\pi \cdot\left(r_{i}^{2} \cdot h_{i}-r_{p}^{2} \cdot h_{p}\right) \cdot \rho \cdot \Delta H}{t},
\end{aligned}
$$

where $h_{a}$ is the average convection heat transfer coefficient on the surface in $\mathrm{W} / \mathrm{m}^{2} \cdot{ }^{\circ} \mathrm{c} ; A_{o}$ is the outside surface area of cylinder model in $\mathrm{m}^{2} ; \varepsilon$ is the dimensionless surface's emissivity of insulating material; $\sigma=5.670 \times 10^{-8} \mathrm{~W} / \mathrm{m}^{2} \cdot \mathrm{K}^{4}$ is the Stefan-Boltzmann constant; $\Delta H$ is the latent heat of $\mathrm{PCM}$ in $\mathrm{J} / \mathrm{kg} ; \rho$ is the density of the PCM in $\mathrm{kg} / \mathrm{m}^{3} ; r_{p}$ and $h_{p}$ are the radius and height of the product (The principle of this transition is volume equaling) in $m$; $t$ is duration time of package in $s ; k$ is the thermal conductivity of the insulating material in $\mathrm{W} / \mathrm{m} \cdot{ }^{\circ} \mathrm{C} ; r_{i}$ and $r_{o}$ are the inside and outside radii of cylinder model in $m ; h_{i}$ and $h_{o}$ are the inside and outside heights of cylinder model in $m$.

Actually, $r_{i}$ expresses not only the inside radius of model, but also the dosage of phase change material. The bigger the inside radius of cylinder model is, the more PCM is used.

From (4), the following relation among parameters of cylinder model, ambient and target temperature, size of product, and duration time of package can be achieved

$$
\begin{aligned}
& 2 r_{o} \cdot\left(r_{o}+h_{o}\right) \\
& \cdot\left[h_{a}\left(T_{\infty}-\frac{\left(r_{i}^{2} \cdot h_{i}-r_{p}^{2} \cdot h_{p}\right) \cdot \rho \cdot \Delta H}{2 \cdot k \cdot t \cdot\left(\left(r_{o}^{2} / \Delta x\right)+\left(h_{o} / \ln \left(r_{o} / r_{i}\right)\right)\right)}-T_{i}\right)\right. \\
& \left.+\varepsilon \cdot \sigma\left(T_{\infty}^{4}-\left(\frac{\left(r_{i}^{2} \cdot h_{i}-r_{p}^{2} \cdot h_{p}\right) \cdot \rho \cdot \Delta H}{2 \cdot k \cdot t \cdot\left(\left(r_{o}^{2} / \Delta x\right)+\left(h_{o} / \ln \left(r_{o} / r_{i}\right)\right)\right)}+T_{i}\right)^{4}\right)\right] \\
& =\frac{\left(r_{i}^{2} \cdot h_{i}-r_{p}^{2} \cdot h_{p}\right) \cdot \rho \cdot \Delta H}{t} .
\end{aligned}
$$

It is especially to point out that when shipping time, target temperature of product, size of product, and type of PCM and insulated material were given, a curve of $r_{i}-\Delta x(\Delta x=$ $r_{o}-r_{i}$ ) could be achieved. Each point on the curve represents a design solution for insulating packaging, which means the geometry dimensions of container and dosage of PCM based on each point could maintain product in target temperature during required shipping time.

3.2. Insulating Packaging Solution Based on Cylinder Model. Based on the demands of a package (such as ambient temperature, shipping time, and size of product), the designer can apply the curve of $r_{i}-\Delta x$ (inner radius-thickness on cylinder model) for a set of given inputs from (5). In this case the designer has many options for inside radiuses and thicknesses. Each combination offers different package solutions which can satisfy all the basic given requirements. However, if the designer wants to determine a single optional solution in terms of a set of specifics, "the minimum cost" rule could be used as the optimized target. Lowest cost is the ultimate goal of the designer when the package can meet all of packaging functions.

To reduce the cost of packaging is to reduce the cost of the package itself. In insulating packages, the packaging cost includes the cost of the packaging container and the PCM. In this case, the cost of the package can be expressed as follows:

$$
\begin{aligned}
\text { Cost } & =\text { Cost of Container }+ \text { Cost of PCM } \\
& =\text { Cylinder Mass } \times A+\text { PCM Mass } \times B \\
& =\pi \cdot\left(r_{o}^{2} h_{o}-r_{i}^{2} h_{i}\right) \cdot \rho_{1} \cdot A-\pi \cdot r_{i}^{2} h \cdot \rho \cdot B,
\end{aligned}
$$

where $A$ is the cost of insulated material per unit mass. $B$ is the cost of PCM per unit mass. $\rho_{1}$ and $\rho$ are the density of the insulated material and PCM, respectively.

Therefore, to have an insulating package design, the procedure is as follows.

(1) Start from the curve of the inside radius-thickness of cylinder model $\left(r_{i}-\Delta x\right)$. Equation (5) provides the relationship between the inside radius $\left(r_{i}\right)$ and the thickness of cylinder model $(\Delta x)$ when both outside convection and radiation are considered.

(2) Determine related parameters from the inputs in (5). To shorten the experiment time, assume the duration of the package is 24 hours (like domestic shipping). All parameters in the equation are shown as follows:

$$
\begin{gathered}
t=24 \text { hours; } \quad T_{\infty}=50{ }^{\circ} \mathrm{C} ; \quad \rho=540 \mathrm{~kg} / \mathrm{m}^{3} ; \\
h_{o}=h_{i}+2 \Delta x=0.4 \mathrm{~m} ; \quad L_{p}=W_{p}=0.1 \mathrm{~m} ; \\
\quad h_{p}=0.2 \mathrm{~m} ; \quad \Delta H=335000 \mathrm{~J} / \mathrm{kg} ; \\
k=0.039 \mathrm{~W} / \mathrm{m} \cdot{ }^{\circ} \mathrm{C} ; \quad h_{a}=1.847 \mathrm{~W} / \mathrm{m}^{2} \cdot{ }^{\circ} \mathrm{C} ; \\
\varepsilon=0.9 ; \quad \sigma=5.67 \times 10^{-8} \mathrm{~W} /\left(\mathrm{m}^{2} \cdot \mathrm{K}^{4}\right) .
\end{gathered}
$$


(3) Generate the curve of the inside radius-thickness of cylinder model $\left(r_{i}-\Delta x\right)$. Figure 4 shows the relationship between the inside radius and the thickness when combination of convection and radiation is considered for the previously given set of inputs.

(4) Determine the design. After setting the optimizing target as the minimum cost, an additional relation can be created to determine the design condition on the curve. As indicated in the reference prices for insulated containers (such as EPS foam) on the website, the price of containers is $¥ 40$ yuan $/ \mathrm{kg}$, and the price of broken ice is $¥ 14$ yuan $/ \mathrm{kg}$. Applying all the data to (6), the cost-thickness curve based on Figure 4 was shown in Figure 5. It shows that the minimum cost of package is $¥ 20.97$ yuan when the thickness of package is $34 \mathrm{~mm}$, and the inside radius of cylinder model is $113 \mathrm{~mm}$ from the given cost information of the materials. In this case, the quantity of ice (PCM) can be calculated by using this equation:

$$
\begin{aligned}
& \pi\left(r_{i}^{2} h_{i}-\left(\sqrt{\frac{L_{p} \times W_{p}}{\pi}}\right)^{2} h_{p}\right) \rho \\
& =\pi \cdot\left(0.113^{2} \cdot 0.332-\frac{0.1 \times 0.1}{\pi} \cdot 0.2\right) \cdot 540000 \\
& =6111.8 \mathrm{~g} .
\end{aligned}
$$

Therefore, not only the design of the package is completed, but also the cost and the quantity of PCMs become available to users.

(5) Return the cylinder model to rectangular container design. It is well known that a cylinder is not practical for packaging applications. The cylinder model only serves as an analog for packaging design. Designers should transform the specific cylinder back to a rectangular container. By using transfer (2), suppose the section of rectangular is square; the inside dimension of rectangular container could be as follows:

$$
L=W=\sqrt{\pi r_{i}^{2}}=\sqrt{\pi \cdot 0.113^{2}}=0.2003 \mathrm{~m} .
$$

Till now, the insulating packaging solution based on required information was achieved: the inside dimensions of an insulating container are $200 \times 200 \times 332 \times 34 \mathrm{~mm}$, the dosage of PCM (ice) is $6111.8 \mathrm{~g}$, and total cost of each packaging system is $¥ 20.97$ yuan.

\section{Verification Test}

To verify this optimized result, an experiment needs to be set up for the final insulating packaging system. A custom insulating container was made on the basis of the packaging solution. To simplify the manufacture, the inside dimensions of final container are $200 \times 200 \times 332 \mathrm{~mm}$, and its thickness is $34 \mathrm{~mm}$.

A verification test was run on the custom rectangular insulating container, with loading the calculated amount of

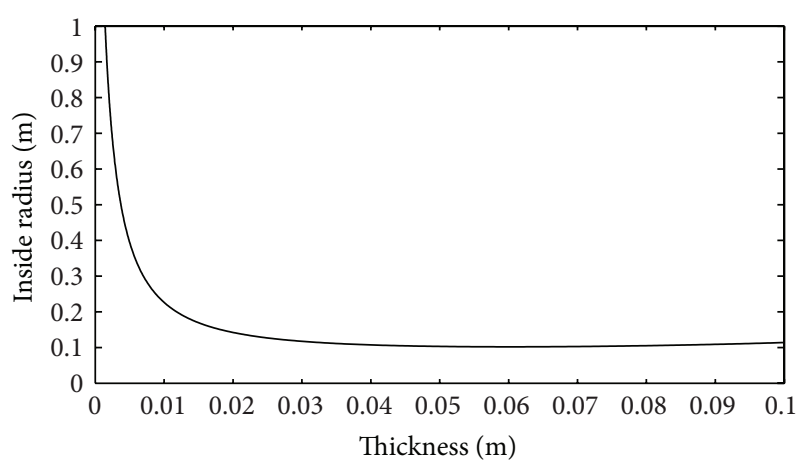

FIGURE 4: Inside radius of cylinder thickness of wall curve on package solution.

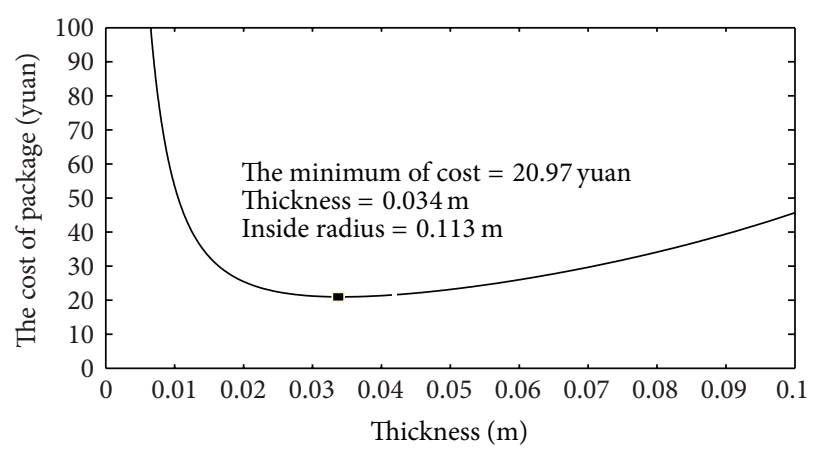

FIgURE 5: Cost-thickness curve.

PCM (6111.8 g broken ice) into the container as a packaging prototype. Setting up the experiment with ambient temperature $\left(50^{\circ} \mathrm{C}\right)$, the test data were obtained in Figure 6 . The duration time of the packaging system is selected to verify the developed cylinder model.

Figure 6 shows that the duration of the package is 25 hours and 21 minutes. The duration of the package in the experiment is 81 minutes longer than expected duration time. The error of duration is $6.4 \%$. This demonstrates that the insulating package design based on cylinder model is successful.

\section{Conclusions and Recommendations}

In the research, a cylinder model has been developed to simplify heat transfer analysis and the design of an insulating package. The cylinder model provides a working foundation for such a package design by transforming a rectangular insulated container to a cylinder configuration, and vice versa.

Applying the "minimum cost" principle, a designer can decide the size of insulated container, the amount of PCM, and the configuration of the packaging system from the general formulation of the solution of the cylinder model. As a result, this research work offers not only an effective mathematical model for the insulating packaging system, but also a unique transformation between the cylinder and rectangular configurations, providing a straightforward tool, 


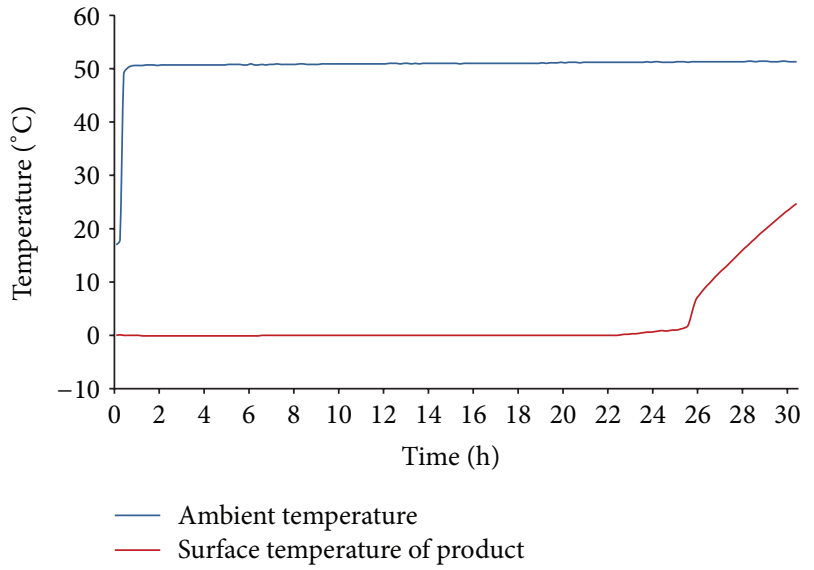

FIGURE 6: Inside and ambient temperatures on validation experiment.

validated by benchmark experiments, making insulating packaging design for the packaging industry.

\section{Acknowledgment}

This research was supported in part by a Grant-in-Aid for Scientific Research on "The Mechanism Research on Insulating Packaging Design" from the Ministry of Education of China.

\section{References}

[1] S. P. Singh, G. Burgess, and J. Singh, "Performance comparison of thermal insulated packaging boxes, bags and refrigerants for single-parcel shipments," Packaging Technology and Science, vol. 21, no. 1, pp. 25-35, 2008.

[2] S.-J. Choi and G. Burgess, "Practical mathematical model to predict the performance of insulating packages," Packaging Technology and Science, vol. 20, no. 6, pp. 369-380, 2007.

[3] L. J. Stavish, "Designing insulated packaging for perishable in vivo diagnostics," Medical Device and Diagnostic Industry, vol. 6, no. 8, pp. 105-108, 1984.

[4] S. Cook, "Thermo of Packaging," Pharma/Device Packaging, pp. 26-28, 1999.

[5] N. Kositruangchai, Theoretical, experimental and computer model for package R-value using regular ice and dry ice [M.S. thesis], Michigan State University, East Lansing, Mich, USA, 2003.

[6] B. Zalba, J. M. Marín, L. F. Cabeza, and H. Mehling, "Review on thermal energy storage with phase change: materials, heat transfer analysis and applications," Applied Thermal Engineering, vol. 23, no. 3, pp. 251-283, 2003.

[7] K. Motsunaga, G. Burgess, and H. Lockhart, "Two methods for calculating the amount of refrigerant required for cyclic temperature testing of insulated packages," Packaging Technology and Science, vol. 20, no. 2, pp. 113-123, 2007.

[8] J. Qian and J. Mo, "Insulating packaging solutions based on spherical shell model," International Journal of Comprehensive Engineering C, vol. 1, pp. 50-57, 2012.
[9] Y. Zhao and J. Qian, "The development of insulating packaging based on cylinder model," Packaging Engineering, vol. 33, no. 9, pp. 18-22, 2012. 


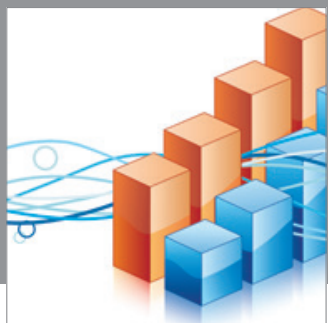

Advances in

Operations Research

mansans

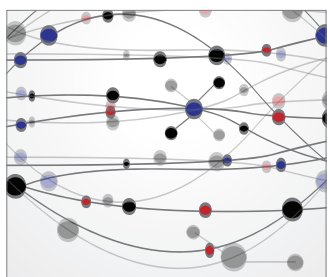

The Scientific World Journal
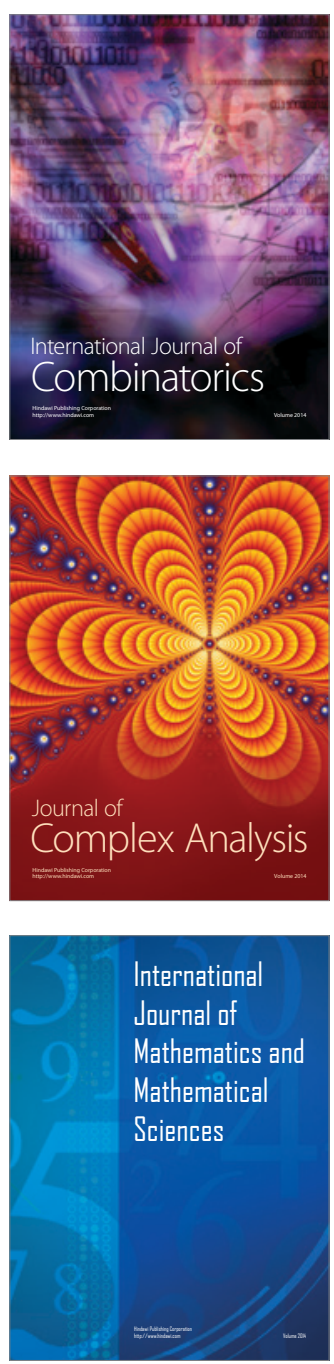
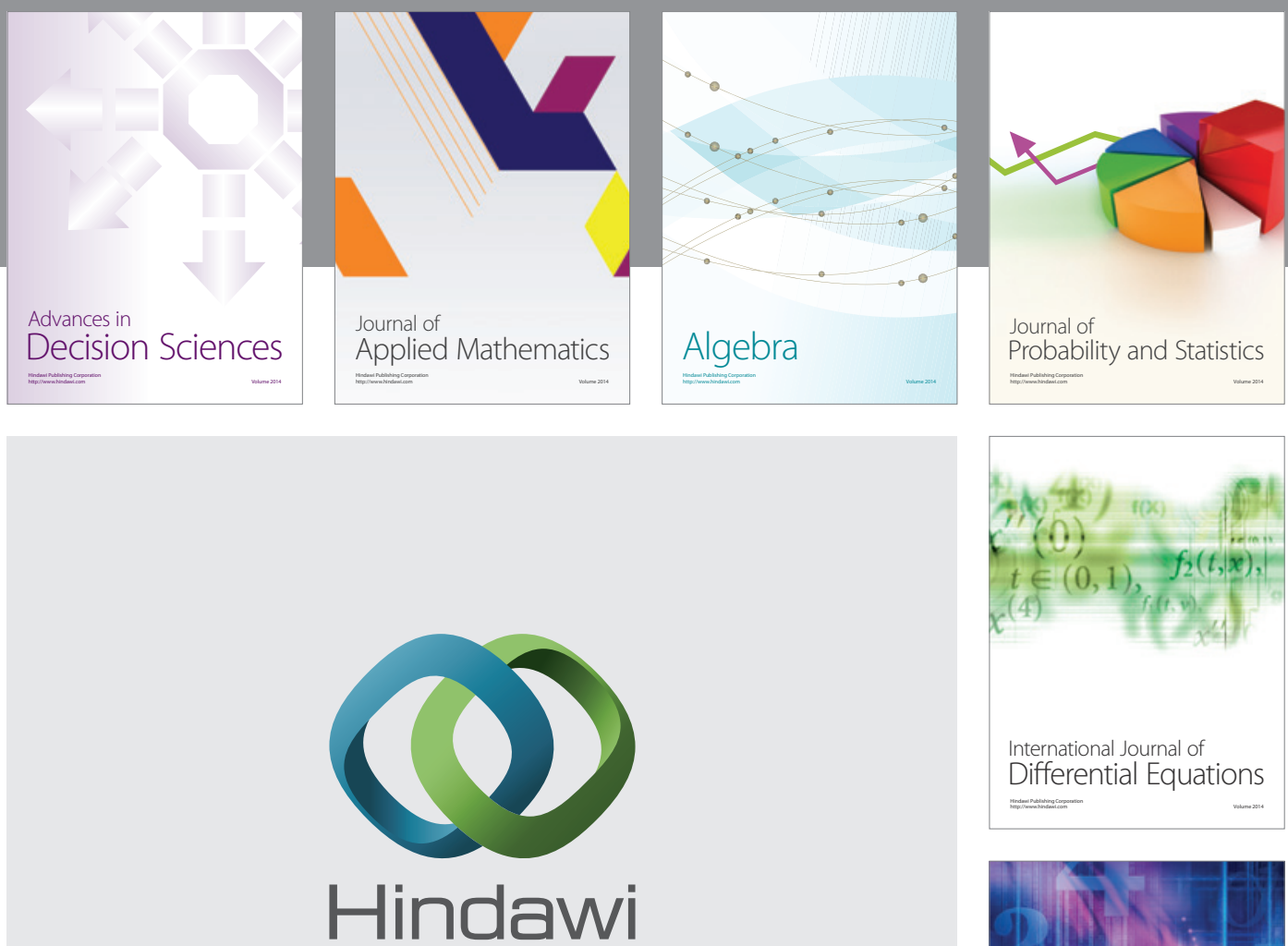

Submit your manuscripts at http://www.hindawi.com
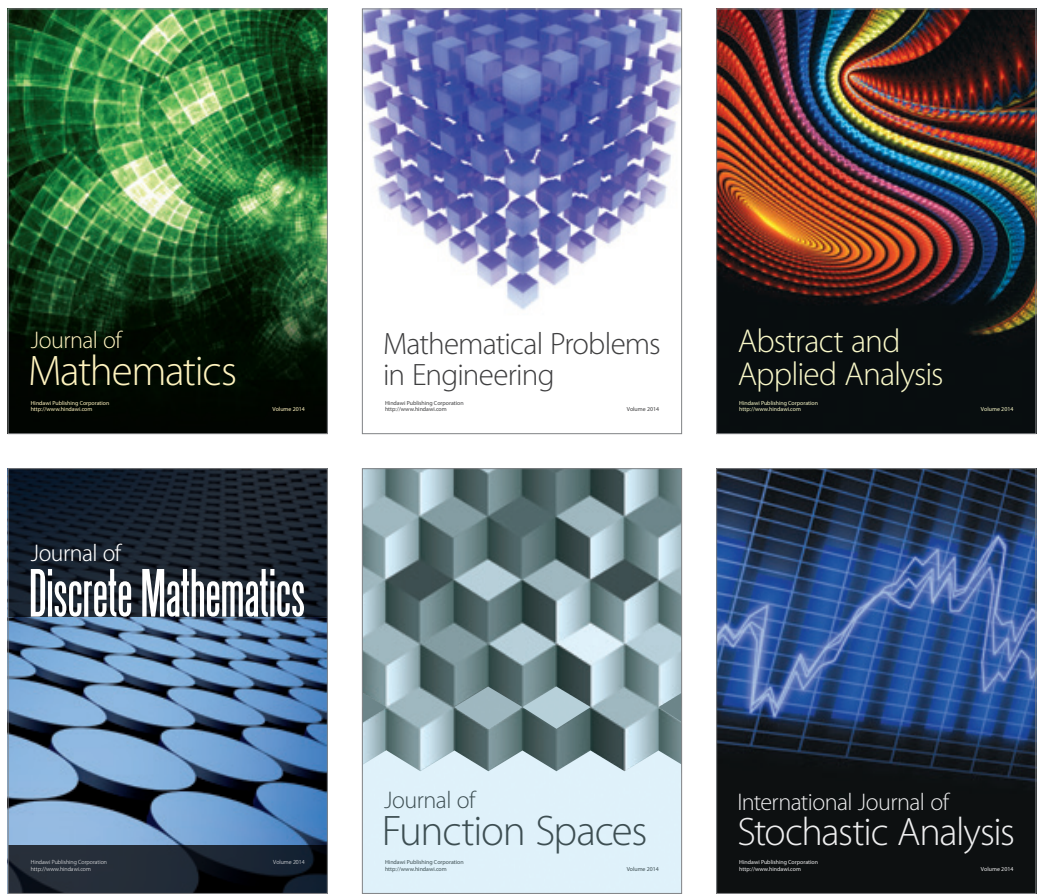

Journal of

Function Spaces

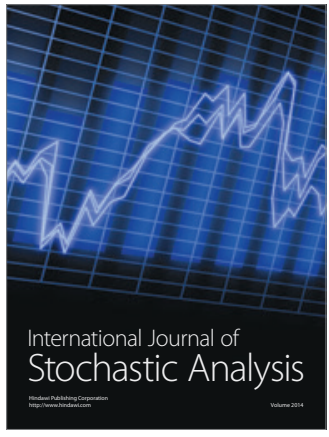

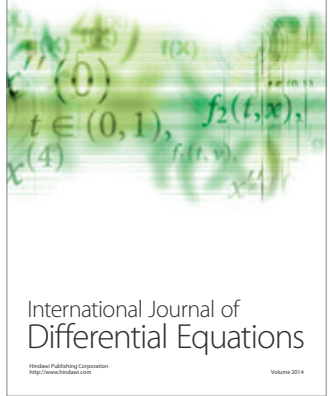
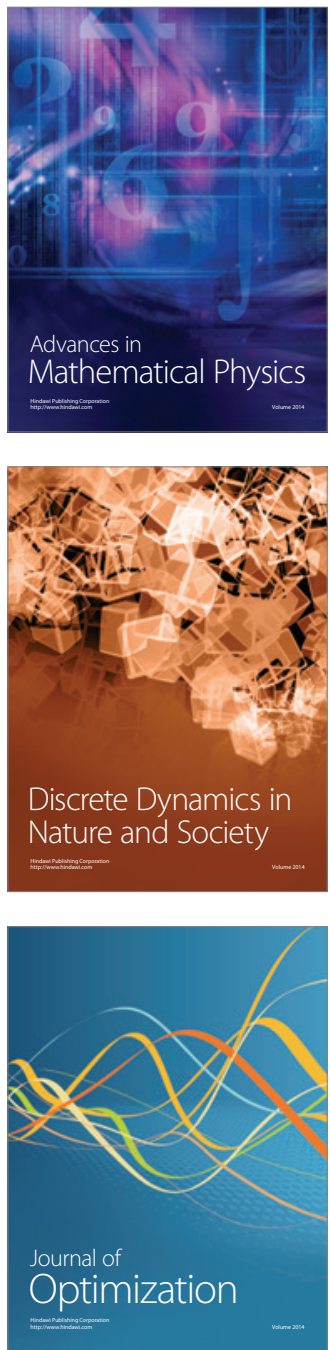